\title{
CRITICAL INSIGHT
}

\section{Do Charge State Signatures Guarantee Protein Conformations?}

\author{
Zoe Hall, Carol V. Robinson
}

Department of Chemistry, Physical and Theoretical Chemistry Laboratory, University of Oxford, South Parks Road, Oxford, OX1 3QZ, UK

\begin{abstract}
The extent to which proteins in the gas phase retain their condensed-phase structure is a hotly debated issue. Closely related to this is the degree to which the observed charge state reflects protein conformation. Evidence from electron capture dissociation, hydrogen/deuterium exchange, ion mobility, and molecular dynamics shows clearly that there is often a strong correlation between the degree of folding and charge state, with the most compact conformations observed for the lowest charge states. In this article, we address recent controversies surrounding the relationship between charge states and folding, focussing also on the manipulation of charge in solution and its effect on conformation. 'Supercharging' reagents that have been used to effect change in charge state can promote unfolding in the electrospray droplet. However for several protein complexes, supercharging does not appear to perturb the structure in that unfolding is not detected. Consequently, a higher charge state does not necessarily imply unfolding. Whilst the effect of charge manipulation on conformation remains controversial, there is strong evidence that a folded, compact state of a protein can survive in the gas phase, at least on a millisecond timescale. The exact nature of the side-chain packing and secondary structural elements in these compact states, however, remains elusive and prompts further research.
\end{abstract}

Key words: Protein charge state, Gas-phase structure, Supercharging

\section{Introduction}

$\mathrm{O}$ ver the past two decades, since the introduction of electrospray [1] and the first experiments to demonstrate the preservation of noncovalent binding in the mass spectrometer [2], one of the most controversial issues has been the extent to which gas-phase protein structures mirror those found in solution. This has come to the fore with several recent publications. The first publication involves an intriguing article in which the authors present evidence for preservation of folded

Electronic supplementary material The online version of this article (doi:10.1007/s13361-012-0393-z) contains supplementary material, which is available to authorized users.

Correspondence to: Carol Robinson; e-mail: carol.robinson@chem.ox.ac.uk structure following femtosecond laser vaporization with electrospray post-desorption [3]. The authors use the charge state signature of the protein to report on their conformation following laser vaporization, and compare the results of these experiments with 'conventional' electrospray. The second controversy is an on-going debate discussed in several publications, which again relates to the relationship between the charge state and protein conformation. This time, the debate centers on the extent to which charge manipulation, either supercharging or charge reduction, affects the folded structure within a protein or protein complex. In this article, we consider the experimental evidence for the assertion that folding is reflected in the charge state distribution and consider how charge manipulation affects the folded state.

Dealing first with the controversy that folded structure is retained by proteins in the gas phase, a strong counter 
argument to this statement is proposed by McLafferty and colleagues stating that folded protein in the gas phase is 'an unproven idea' [4]. Citing previous studies, evidence is presented that gas-phase proteins can unfold and reorganize $[5,6]$. In a response to this correspondence, Levis and colleagues state that the ambiguity arising between the two viewpoints is due to the precise definition of gas-phase protein ions [7]. Levis and colleagues denote this as protein that has transferred into the gas phase, presumably with water molecules still attached, while for McLafferty and colleagues this state is attributed to that of the protein after complete desolvation. In this article, we consider the experimental evidence from both groups and supplement this with additional data from ion mobility (IM) experiments, gasphase hydrogen/deuterium exchange reactions, studies of noncovalent complexes, and molecular dynamics (MD) simulations.

\section{How Folded is a Gas-Phase Protein - Evidence from ECD Experiments and MD Simulations}

First we consider the seminal papers of Breuker and McLafferty, in which native electron capture dissociation (NECD) of protein ions is used to probe folded structure of cytochrome $c[8,9]$. Such experiments exploit the fact that protein backbone cleavage occurs exclusively next to residues that are in contact with an electron donor (the heme group). Site-specific thermal unfolding in the gas phase, which results in separation of residues from the heme group, is monitored by the decrease in the corresponding fragments in the NECD spectra. Using this approach to probe the structure of cytochrome $c$ after transfer into the gas phase, the unfolding of regions interacting hydrophobically in the native structure could be shown, despite the fact that these regions were sometimes the last to unfold in solution [10]. The results of these experiments were supported by MD simulations of cytochrome $c$, surrounded by a monolayer of water and placed in a vacuum [11]. The simulations are consistent with evaporation of water from the native protein, with the last water molecules to leave being those which had been attached to the ionic side chains [5]. Complete loss of water leads to the collapse of exterior ionic groups, which form new interactions with each other, protecting native-like interactions within the protein ion. Storage of ions for millisecond time periods can result in loss of hydrophobic and electrostatic interactions. MD simulations imply that transient unfolding could facilitate formation of new noncovalent interactions within a protein, producing more stable gas-phase structures [5].

Recently, further experimental evidence from ECD was presented in an investigation of a three-helix bundle protein KIX [12]. These ECD experiments rely on unselective backbone cleavage, with the retention of noncovalent intramolecular interactions, which prevent the separation of backbone cleavage products. In this way, the abundance of ECD fragments provides a measure of the extent of gasphase unfolding. The experimental data showed the most stable regions of the protein to be those forming salt bridges and ionic hydrogen bonds [12]. The wider implications of this study of KIX are that if proteins are stabilized by a favorable network of electrostatic interactions, their native structure is more likely to survive in the gas phase than a protein with less electrostatic interactions.

\section{Can Charge State Signatures be Used as Evidence of Folding?}

Having established evidence for unfolding and for the preservation of native structure where electrostatic interactions predominate, we now turn to the recent paper that brought the folded structure in the gas phase controversy to a head. Levis and colleagues have described a femtosecond laser vaporization method in which folded protein structure is thought to be conserved [3]. In these experiments, a protein-containing solution, placed on a steel sample plate, is vaporized by a laser pulse. The vaporized proteins are then subjected to electrospray [13]. The major advance claimed is that proteins vaporized by a femtosecond laser can retain a folded structure during this process. This observation is rationalized since the energy from the laser is deposited much faster than that required to produce unfolding or reorganization of protein structure [3]. The authors use the charge state signature of the protein to conclude that the folded conformation is retained during, and immediately after, laser desorption. The possibility that the proteins are able to refold in the electrospray buffer cannot be discounted. However, if correct, this technique would certainly be important for ionizing proteins and their complexes directly from their native environment, without the need for lengthy separation and isolation protocols.

The interpretation of the data and the ensuing controversy lie not just in the definition of the dehydrated state of the protein, as suggested by the Levis group [7], but rather in how precisely the charge state distribution represents the folded state of the gas-phase protein. This approach, first suggested by Chait and colleagues, to follow heat-induced unfolding of a number of proteins including ubiquitin, cytochrome $c$, and lysozyme, showed that higher charge states were correlated with thermal unfolding, effected in a stainless steel capillary immediately prior to electrospray [14]. Early work, also from Chait, demonstrated that the observed charge state signature was linked to the presence of anions in solution [15] and held the cautionary note that attempts to correlate the distribution of charge states observed on proteins in the gas phase with the net charge residing on the protein in solution will require that the anion effect be taken into account. To consider the relationship between charge state and folded protein further, we need to look at other experimental evidence. 


\section{Evidence from Gas-Phase Hydrogen/Deuterium Exchange Experiments and Ion Mobility}

In addition to the charge state signature and ECD experiments there are alternative means of assessing the folded states of proteins in the gas phase. These include hydrogen/deuterium exchange (HDX) in the gas phase, which reports on protection from exchange and provides a read out of the number of exchangeable sites involved in hydrogen bonding interactions. Pioneering experiments, again by the McLafferty group, showed that labile protons in cytochrome $c(6+$ to $17+)$ were exchanged for deuterium, in different patterns consistent with at least six gas-phase conformations [16]. Infrared laser heating and fast collisions were found to induce ions to unfold and exchange to a greater extent, while charge-stripping ions to lower charge values yielded apparent folding. These early experiments support the link between charge state and accessible protons in the gas phase but cannot report directly on the overall packing of the secondary structural elements within the protein.

IM enables measurement of collision cross sections (CCS), which can then be compared with CCS calculated for the native state or partially folded and fully unfolded states. Measurement of drift times as a function of charge state was used to probe the gas-phase structure of cytochrome $c$ ions [6]. Increases in the charge state of the protein ions were found to correlate with an increase in CCS, beyond that calculated for the native state. This is consistent with the unfolding of the more highly charged ions in the gas phase, although this could also result from unfolding in the solution conditions used to generate these high charge states (Figure 1a). Moreover, storage of these gas-phase protein ions was also shown to lead to an increase in CCS [17]. Combining HDX with IM-MS to probe the gas-phase structure suggests that the 5+ charge state of cytochrome $c$ ions have near native-like structure, whereas the $9+$ charge state contains an extended helix [18].

Further support for compact structure of low charge states comes from recent IM-MS studies of ubiquitin. Solutions that stabilize the native state of ubiquitin yielded essentially one family of tightly folded desolvated structures with CCS that match the size of the native structure [19]. By contrast, solution conditions favoring the partially folded A state yielded CCS matching in size to conformations between the A state and the fully unfolded state. The A state, which is charged more extensively than the native-like state, decays rapidly $(\leq 50 \mathrm{~ms})$, unfolding to more extended structures. By contrast, the native-like conformation of ubiquitin survives for $>100 \mathrm{~ms}$ in a $294 \mathrm{~K}$ solvent-free environment [19]. These experiments establish that compact structures for low charge states can survive for extended periods of time in the gas phase.

Interestingly, the results from these recent experiments from the Bowers group, where unfolding of the native state is not observed upon transfer into the gas phase, are at odds with earlier studies [20] where extensive unfolding of $\sim 50 \%$ of ubiquitin $7+$ ions was observed after $\sim 200 \mathrm{~ms}$. This apparent discrepancy prompted further investigation with very recent ECD experiments of ubiquitin [21]. The results of these ECD experiments show that the proportion of elongated structures after desolvation was lower than that found in the earlier study [20] but greater than that observed by Bowers and co-workers [19]. While many of the differences in these studies can be attributed to different experimental setups and solution conditions, the important point of the ECD experiments is to establish that the order of unfolding of the various regions is dictated by the extent to which hydrophobic bonding is compensated for by electrostatic interactions [21]. Together, these ECD and IM experiments establish a relationship between the charge state of the protein and the extent of unfolding in the gas phase, with higher charge states experiencing unfolding to a greater extent, and demonstrate the importance of electrostatic interactions in stabilising gas-phase structures.

\section{Can Charge States be Manipulated Without Affecting Folded Protein Structure?}

Turning now to the second controversy: the use of solution additives to manipulate charge. While it is clear that charge can be manipulated, it is not clear how these reagents affect the folded structure of a protein or complex. These experiments take the form of 'supercharging' or 'charge reduction' depending on the solution additives. Considering first the evidence for folded structure under supercharging conditions, addition of $m$-NBA or sulfolane $[22,23]$ is used to produce higher charge states than normally accessible by globular proteins and their complexes. An increase in average charge state is reported, from $10+$ to $15+$, for the $29 \mathrm{kDa}$ carbonic anhydrase-zinc complex. The fact that this supercharging does not result in loss of protein-ligand interactions [22] is evidence that the ligand binding cavity is retained, implying that the structure is native-like.

A series of experiments seemingly at odds with these results have shown that for the protein holo myoglobin, addition of sulfolane leads to unfolding of the protein as assessed by partial loss of heme binding. Additionally, the stability of myoglobin was followed by circular dichroism spectroscopy whilst titrating with a denaturant, in the presence of sulfolane. This revealed that the native state is destabilized with increasing sulfolane concentration [24]. Moreover supercharging was affected by chemically modifying cytochrome $c$ with up to seven cross-links or ubiquitin with up to two cross-links. While these cross-links did not affect the average charge states of these proteins in the absence of $m$-NBA, the extent of supercharging induced by $m$-NBA increased with decreasing numbers of cross-links [25].

In order to lower the charge state, charge reduction is commonly carried out by the use of solution additives, such 
(a)

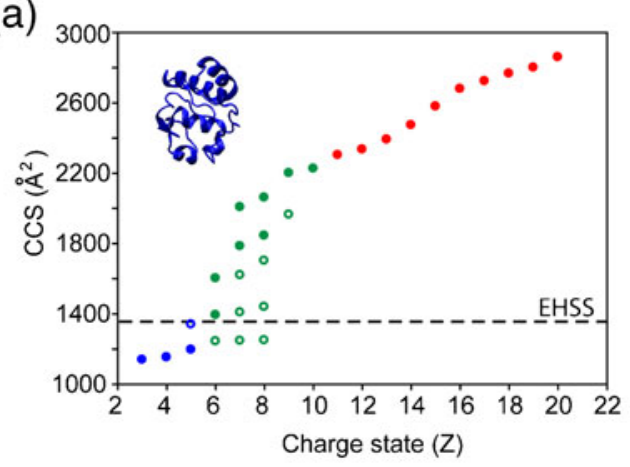

(b)

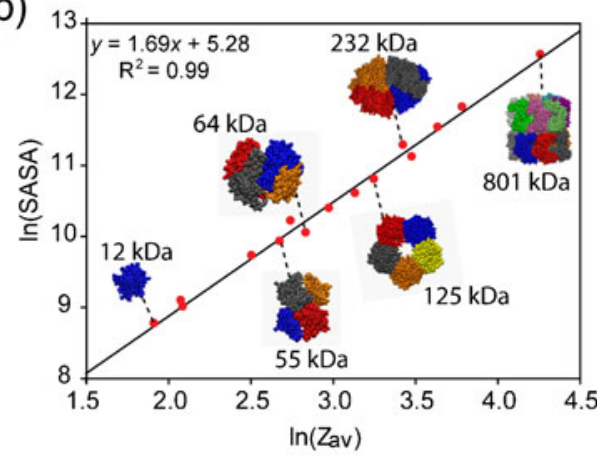

(c)

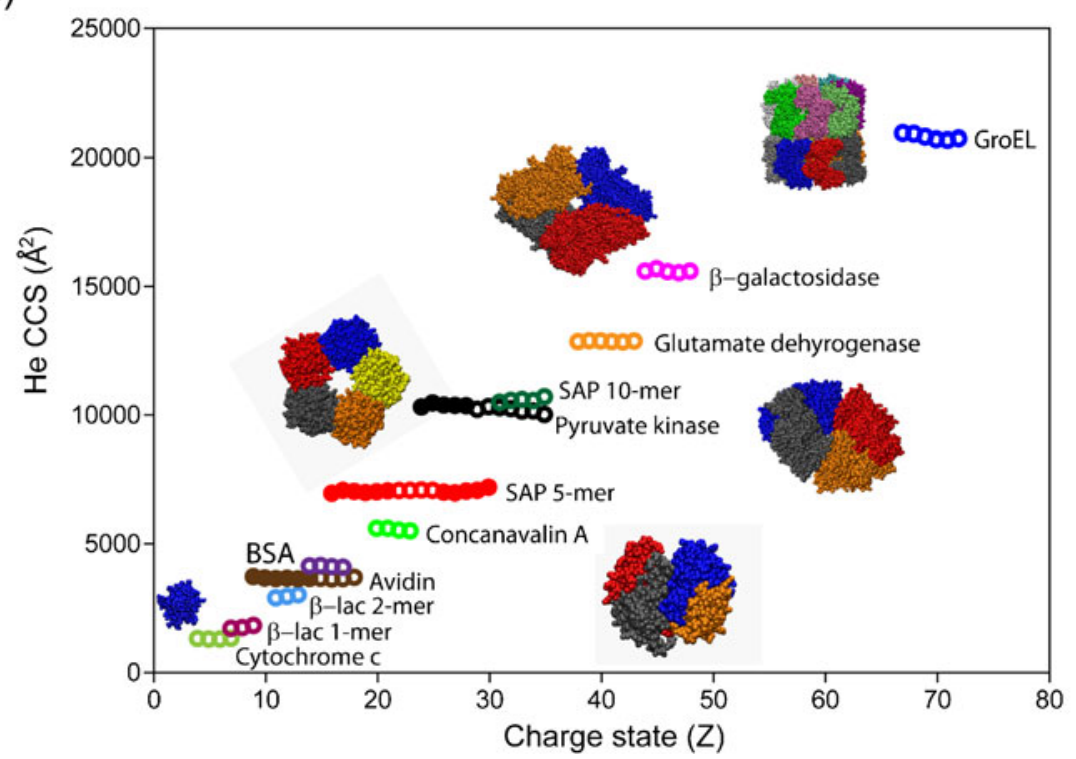

Figure 1. Relationship between charge state and structure (a) CCS of cytochrome $c$ (75:25 water:acetonitrile) is influenced by charge state, with higher charge states occupying more extended conformations. A plot of CCS versus charge state is shown [52]. Charge states 11-20+ (red) were generated by acidifying the solution with $2.5 \%$ acetic acid, whereas charge states $<6+$ (blue) were generated by charge stripping, through the introduction of a base into the desolvation region. The filled and open points show the CCS for the features dominating at high and low injection energies respectively. A dashed line represents the EHSS value for CCS of the native conformation. (b) The average charge state from native MS $\left(Z_{\text {av }}\right)$ can be used to predict protein complex solvent accessible surface area (SASA). Data shown is from a series of proteins and protein complexes electrosprayed in-house from $200 \mathrm{mM}$ ammonium acetate (AA) buffer. These are cytochrome $c$, myoglobin, $\beta$-lactoglobulin (1mer, 2-mer), TTR, BSA, avidin, concanavalin A, SAP (5-mer, 10-mer), ADH, pyruvate kinase, glutamate dehydrogenase, $\beta$ galactosidase, and GroEL, and range in size from 12 to $801 \mathrm{kDa}$. (c) The overall structure of individual proteins and protein complexes in buffered solutions is not generally influenced by charge state, as noted by constant CCS for a wide range of charge states for individual species. Open points show CCS of proteins/complexes, which had been electrosprayed from $200 \mathrm{mM}$ AA [53]. Filled points show additional CCS values for charge reduced avidin, SAP and pyruvate kinase, as well as supercharged SAP. Charge states 9-14+ for avidin, 16-21+ for SAP 5-mer, and 24-28+ for pyruvate kinase, were generated from charge reduction in solution by the addition of 10-20 mM TEA or 20 mM DBU base (avidin 9+). Charge states 26-30+ for SAP 5-mer were generated by the addition of $1 \% \mathrm{~m}$-NBA

as triethylamine (TEA) and imidazole, which have higher gas-phase basicities than typical MS buffers. In the final stages of desolvation, these species effectively compete for charge with ionized sites on the protein. Electrolytes with higher gas-phase basicities will, therefore, remove more charge from the protein [26, 27]. A study in which lysozyme, among others, was progressively charge-reduced from $10+$ to $3+$ showed, by circular dichroism spectroscopy, that there is no loss in secondary structure for any of the charge states [28]. This is in direct contrast to the supercharging experiments, in which it is proposed that the corresponding increase in charge, observed for several proteins, is due in part to unfolding in the final stages of desolvation in the electrospray droplet [24]. 


\section{How Does Charge State Affect Large Protein Complexes?}

It is important to point out that, as proposed previously, there is a strong correlation between the solvent accessible surface area and the average charge state determined experimentally [29, 30] (Figure 1b). For large protein complexes (Figure 1c), experimental evidence supports a mechanism whereby manipulation of surface charge, by charge reduction [31] or enhancement [32], does not necessarily perturb the protein structure as evidenced for transthyretin [31], stable protein 1 [32], and a protective antigen prechannel complex [33]. This is contrary to the situation observed for concanavalin $\mathrm{A}$ in which the tetramer $\mathrm{CCS}$, and solution phase tetramer to dimer equilibrium, was perturbed by the addition of $m$-NBA [33].

To investigate this further, we examined the wellcharacterized pentameric protein complex SAP (125 kDa) for which 14 different charge states were observed. We found that the supercharged species $(26+$ to $30+)$ (Figure 2a) have similar CCS to the complex following electrospray from ammonium acetate buffer $(22+$ to $25+)$ or even in its charge reduced state following addition of TEA $(17+$ to $21+)$ (Figure 2b). The fact that for all 14 charge states, the CCS measurements vary not more than $\pm 1 \%$ provides compelling evidence that the charge on the surface of the complex has little or no effect on its CCS. In this case, monitoring the CCS as a function of activation, it becomes apparent that unfolding of supercharged SAP occurs upon increasing collision energy, in a manner consistent with it proceeding from a folded structure [34] (Figure 2c). Lower charge states at the same laboratory frame energy remain essentially in a compact folded conformation.

Similar experiments were carried out to manipulate the charge states of tetrameric ADH using both TEA and sulfolane to produce 15 different charge states. Charge reduction had very little impact on the overall CCS of the low charge states, while a significant increase was detected for the higher charge states of ADH (Figure 2d). While the behavior of these two different protein complexes, SAP and $\mathrm{ADH}$, is clearly different, it is not yet clear what factors account for the sensitivity to supercharging reagents. It is clear, however, that a large dataset of protein complexes will be needed before conclusions can be drawn as to the whether the preservation of folded structure is dictated by the balance of hydrophobic/hydrophilic residues as in the case of individual proteins discussed above [12].

This balance between hydrophobic and electrostatic interactions was also critical in determining the outcome of early investigations of protein-ligand interactions. For small proteins investigated with a number of ligands, it was often the case that more favorable binding was observed for ionic ligands rather than for hydrophobic ones $[35,36]$. The early observations are, however, particularly interesting in the light of recent observations of hydrophobic ligand binding within an accessible protein cleft [37] and intact membrane protein complexes, wherein the overwhelming number of subunit interactions are hydrophobic [38-40]. In the case of membrane protein subunits, it is tempting to speculate that the micelles used for the introduction of the complex may protect the hydrophobic subunits from the deleterious phase change. However, such an explanation is not possible for the hydrophobic ligand binding interaction and its preservation suggests that a van der Waals component must contribute to the stability of these complexes once in the gas phase. We conclude, therefore, that the importance of electrostatic interactions in determining preservation of the folded state of individual proteins must also be complimented by van der Waals forces when ionic interactions are absent.

\section{Can MD Simulations Inform on Gas-Phase Protein Structure?}

As mentioned above, MD simulations can be particularly informative for exploring structural changes of proteins in the gas phase. Early studies of small proteins [11, 41] and peptides $[42,43]$ have revealed candidate structures for these gas-phase species that have been informative both in terms of folding and the effects of dehydration. In vacuo MD simulations of four globular proteins revealed residual water molecules, aggregating on the protein surface, which assist the preservation of folded structure [44], a result in accord with earlier studies [11]. Limited conformational changes were observed upon dehydration; these included the inward-folding of hydrophilic side chains, which tended to form new hydrogen bonds. In fact, more hydrogen bonds are typically observed in the gas-phase structures than for those same structures in solution [45]. These newly formed hydrogen bonds are likely to play a key role in the stabilization of secondary structure as the protein is transferred into vacuum conditions. Interestingly, similar conclusions were reached through experiment using blackbody infrared radiative dissociation (BIRD) and functional group replacement procedures [46]. In this approach, particular functional groups involved in protein-ligand binding are modified, such that any pre-existing interaction is lost. Comparison of the activation energy of modified and unmodified complex reveals which functional groups are involved in stabilization of the complex. In this way, intermolecular hydrogen bonds within a desolvated proteinligand complex were identified and quantified. Comparison with the crystal structure revealed the partial conservation of intermolecular hydrogen bonds between solution and gasphase structures and identified additional interactions in the gas phase.

In addition to examining the evolution of structure and interactions that soluble proteins undergo in the gas phase, MD simulations have provided support to the protection of membrane protein complexes by micelles during transfer to the gas phase. MD simulations of the membrane protein OmpA171, within a dodecylphosphocholine (DPC) micelle, show the structure of the $\beta$-barrel protein 
(a)

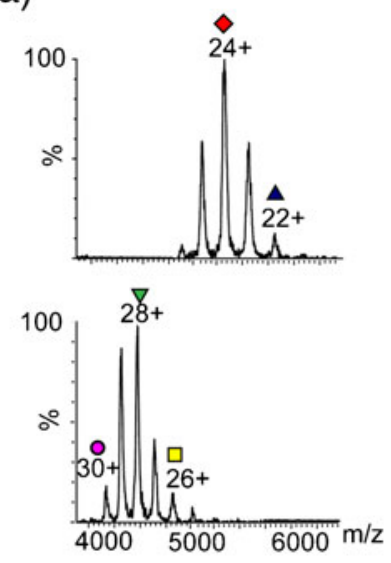

(b)

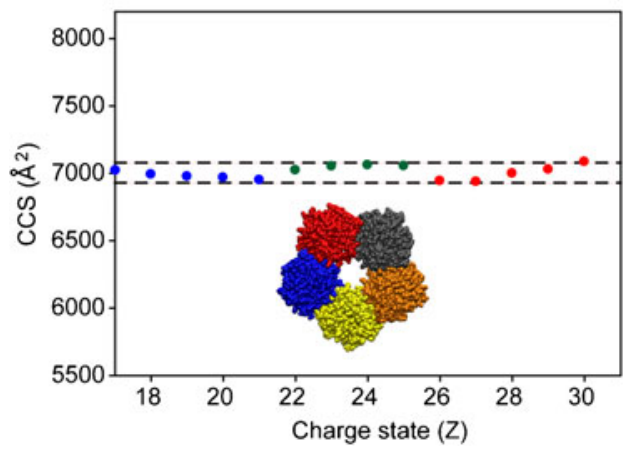

(c)

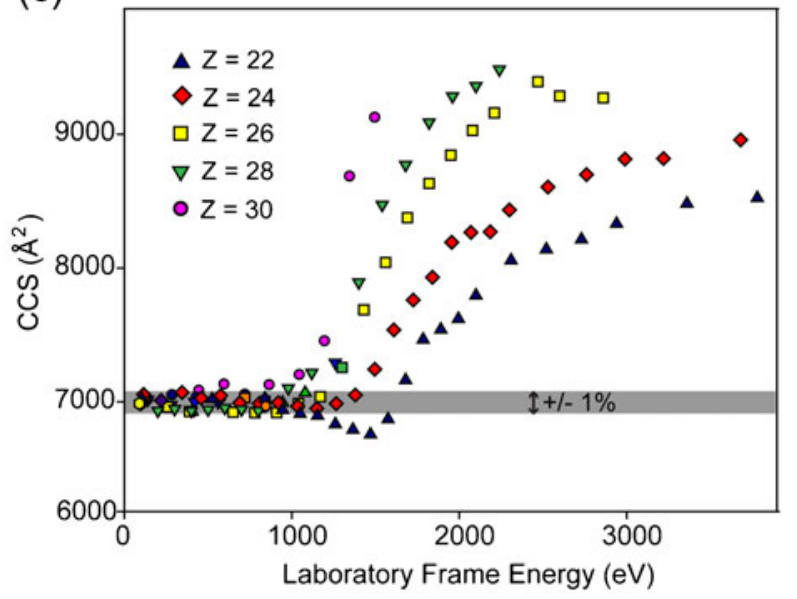

(d)

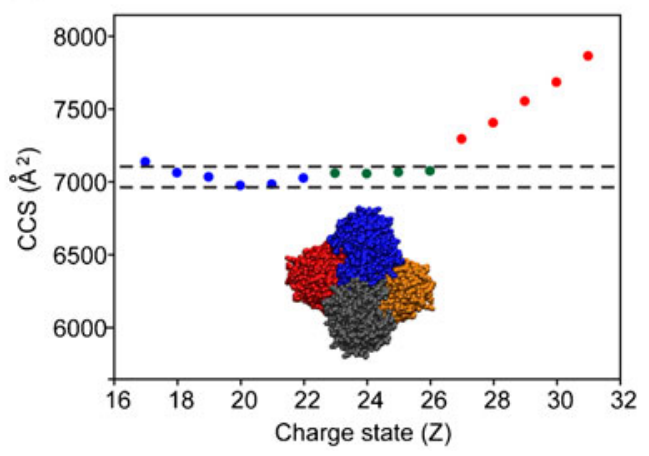

Figure 2. Effect of charge manipulation on folded protein structure. (a) The addition of $1 \% \mathrm{~m}$-NBA to a solution of SAP 5 -mer (125 kDa) in $200 \mathrm{mM}$ ammonium acetate (AA) buffer resulted in the increase of the average charge state from 24+ (upper panel) to 28+ (lower panel). (b) For pentameric SAP, the supercharged species (red) has a similar CCS to the complex following electrospray from AA (green) or triethylamine (TEA) buffer (blue). This is consistent with there being no significant structural changes. Conditions were as follows: 17-21+ (10 mM TEA, $200 \mathrm{mM} \mathrm{AA}), 22-25+(200 \mathrm{mM}$ AA), 26-30+ (1 \% m-NBA, $200 \mathrm{mM}$ AA). The dashed black lines indicate $\pm 1 \%$ from the average CCS of species without supercharging reagent. (c) The CCS of pentameric SAP (22-30+) was monitored, whilst increasing the laboratory frame energy (collision energy $x$ charge). Significant CCS increase, consistent with unfolding, indicates that the structure was initially in a folded state for all charge states [34]. (d) For ADH (143 kDa), the supercharged species (red) have a much greater CCS than that for the complex electrosprayed from AA (green) or TEA buffer (blue). Conditions were as follows: 17-22+ (10 mM TEA, $200 \mathrm{mM} \mathrm{AA}), 23-26+(200 \mathrm{mM} \mathrm{AA}), 27-31+(7 \%$ sulfolane, $200 \mathrm{mM} \mathrm{AA}$ ). The dashed black line indicates $\pm 1 \%$ from the average CCS of species without supercharging reagent

to be virtually unchanged in the gas phase. In order to account for the significantly shorter time scale of the simulation $(150 \mathrm{~ns})$ compared with that of a typical MS experiment (several $\mu \mathrm{s}$ ), simulations were carried out at an elevated temperature $(350 \mathrm{~K})$ [47]. Evidence from these simulations suggests that the micelle does, therefore, shield the native structure during transfer into the gas phase.

Recently, in vacuo MD simulations of a large multisubunit protein complex, pentameric SAP were performed [34]. Through the simulations, we observed the collapse of the ring-topology for low charge states, as the energy of the system was increased. These results were compared with experimental evidence and by manipulation of charge to low charge states. A significant decrease in CCS was observed experimentally, by IM-MS, corresponding closely to col- lapse of the ring-like topology into first a buckled-ring, then finally to a collapsed structure (Figure 3 ). Such a collapse has also been observed previously for virus capsids [48] following collision induced dissociation (CID) and for GroEL [49]. As a further indication of how closely MD simulations can mirror gas-phase experiments, placement of charge asymmetrically between the five subunits of SAP recapitulates the asymmetric dissociation mechanism commonly observed in CID, in which highly charged unfolded monomeric subunits are expelled [34]. This study, therefore, highlights the potential of high temperature vacuum MD simulations of complexes, in combination with experimental data from IM-MS and CID, to provide additional insights into the processes of rearrangement, unfolding, and dissociation in the gas phase. 


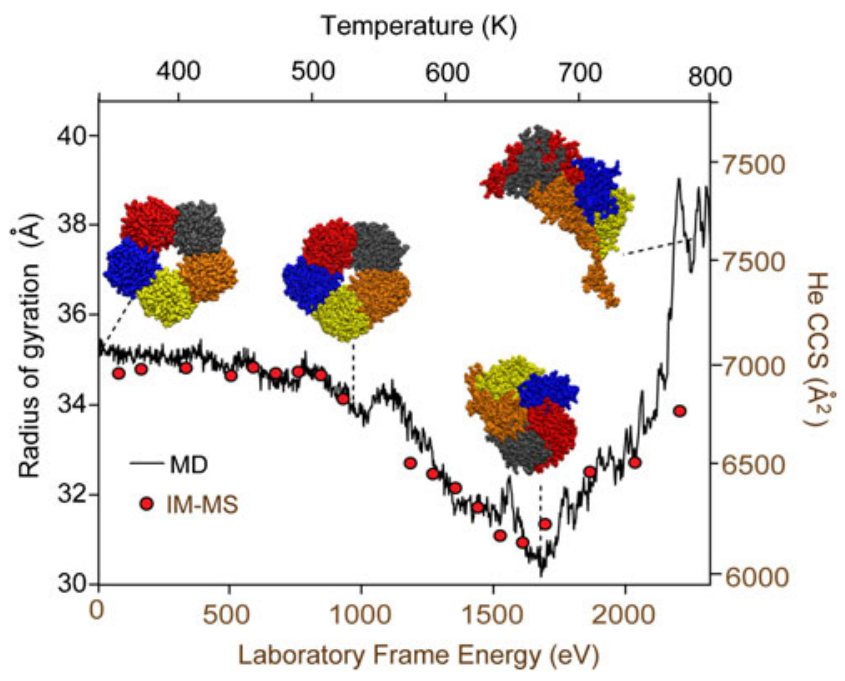

Figure 3. MD simulations and IM-MS follow gas-phase collapse. A combined ion mobility (IM) and molecular dynamics (MD) study [34] followed the collapse of the ringtopology for low charge states of pentameric SAP as the energy of the system was increased. The CCS of SAP 18+ was monitored by IM-MS (red) whilst increasing the laboratory frame energy (collision energy $x$ charge). A significant decrease in CCS was observed, consistent with the formation of a more compact conformation. Similarly, as the temperature was increased from 300 to $800 \mathrm{~K}$ in vacuum simulations, the radius of gyration was monitored (black). Analysis of the simulations showed the collapse of the ringlike topology, before the extension of a monomer

\section{Is There Any Consensus on the Relationship Between Charge and Gas-Phase Protein Structure?}

Consideration of the available evidence from gas-phase HDX reactions, ECD experiments, CCS measurements, and MD simulations points to a consensus that the lowest charge states are the most compact. These low charge states are likely to be the most native-like in terms of secondary structural elements. None of these experiments, however, can report on the precise packing of these structural elements or of the side chain packing of the gas-phase structure; all of these properties are necessary to define the fully folded native state. However, available experimental evidence, together with the MD simulations, strongly implies that a folded, compact state of a protein can survive in the gas phase. Moreover, the fact that a membrane protein complex, with seemingly unfavorable hydrophobic interactions, can survive in the gas phase, points to the preservation of folded structure. Whether or not these have the precise identity of the native state remains to be seen.

This discussion on protein charge states and their link to gas-phase protein structure is unlikely to resolve either of the current controversies about the folded nature of gas-phase proteins and the correlation with their charge state signatures or the effects of charge manipulation on folded protein structure. Pertinent to such a discussion are the recent insights into the electrospray mechanism for proteins $[50$, 51] and macromolecular complexes in which emission of charge carriers was recently proposed to affect the overall charge [26]. While the electrospray mechanism is beyond the scope of the current article, we hope that this discussion will stimulate further research into the influence of charge state on protein unfolding, the chemical reactions that take place in the electrospray droplet, and the extent to which gas-phase proteins unfold during their lifetime.

\section{Acknowledgments}

The authors thank Nina Morgner and Helena Hernández for critical reading of the manuscript. C.V.R is a Royal Society Professor.

\section{References}

1. Fenn, J.B.; Mann, M.; Meng, C.K.; Wong, S.F.; Whitehouse, C.M.: Electrospray ionization for mass spectrometry of large biomolecules. Science 246, 64-71 (1989)

2. Katta, V.; Chait, B.T.: Observation of the heme-globin complex in native myoglobin by electrospray ionization mass spectrometry. J. Am. Chem. Soc. 113, 8534-8535 (1991)

3. Brady, J.J.; Judge E.J.; Levis, R.J.: Nonresonant femtosecond laser vaporization of aqueous protein preserves folded structure. Proc. Natl. Acad. Sci. U.S.A. 108, 12217-12222 (2011)

4. Breuker, K.; Skinner, O.S.; McLafferty, F.W.: Femtosecond laser vaporization that preserves protein-folded structure: An unproven idea. Proc. Natl. Acad. Sci. U.S.A. 109, E206 (2012)

5. Breuker, K.; McLafferty, F.W.: Stepwise evolution of protein native structure with electrospray into the gas phase, $10^{-12}$ to $10^{2}$ s. Proc. Natl. Acad. Sci. U.S.A. 105, 18145-18152 (2008)

6. Clemmer, D.E.; Hudgins, R.R.; Jarrold, M.F.: Naked protein conformations: Cytochrome $\mathrm{c}$ in the gas phase. J. Am. Chem. Soc. 117, 10141-10142 (1995)

7. Brady, J.J.; Judge, E.J.; Levis, R.J.: Reply to Breuker et al.: How laser electrospray mass spectrometry (LEMS) measures condensed phase protein structure, not vacuum structure Proc. Natl. Acad. Sci. U.S.A. 109, E207 (2012)

8. Breuker, K.; McLafferty, F.W.: Native electron capture dissociation for the structural characterization of noncovalent interactions in native cytochrome c. Angew. Chem. Int. Ed. 42, 4900-4904 (2003)

9. Breuker, K.; McLafferty, F.W.: The thermal unfolding of native cytochrome $\mathrm{c}$ in the transition from solution to gas phase probed by native electron capture dissociation. Angew. Chem. Int. Ed. 44, 4911-4914 (2005)

10. Steinberg, M.Z.; Elber, R.; McLafferty, F.W.; Gerber, R.B.; Breuker, K.: Early structural evolution of native cytochrome $c$ after solvent removal. ChemBioChem. 9, 2417-2423 (2008)

11. Steinberg, M.Z.; Breuker, K.; Elber, R.; Gerber, R.B.: The dynamics of water evaporation from partially solvated cytochrome $c$ in the gas phase. Phys. Chem., Chem. Phys. 9, 4690-4697 (2007)

12. Breuker, K.; Bruschweiler, S.; Tollinger, M.: Electrostatic stabilization of a native protein structure in the gas phase. Angew. Chem. Int. Ed. 50, 873-877 (2011)

13. Judge, E.J.; Brady, J.J.; Levis, R.J.: Mass analysis of biological macromolecules at atmospheric pressure using nonresonant femtosecond laser vaporization and electrospray ionization. Anal. Chem. 82, 10203-10207 (2010)

14. Mirza, U.A.; Cohen, S.L.; Chait, B.T.: Heat-induced conformational changes in proteins studied by electrospray ionization mass spectrometry. Anal. Chem. 65, 1-6 (1993)

15. Mirza, U.A.; Chait, B.T.: Effects of anions on the positive ion electrospray ionization mass spectra of peptides and proteins. Anal. Chem. 66, 2898-2904 (1994) 
16. Wood, T.D.; Chorush, R.A.; Wampler, F.M.; Little, D.P.; O'Connor, P.B.; McLafferty, F.W. Gas-phase folding and unfolding of cytochrome c cations. Proc. Natl. Acad. Sci. U.S.A. 92, 2451-2454 (1995)

17. Badman, E.R.; Myung, S.; Clemmer, D.E.: Evidence for unfolding and refolding of gas-phase cytochrome c ions in a paul trap. J. Am. Soc. Mass Spectrom. 16, 1493-1497 (2005)

18. Valentine, S.J.; Clemmer, D.E.: Temperature-dependent H/D exchange of compact and elongated cytochrome c ions in the gas phase. $J$. Am. Soc. Mass Spectrom. 13, 506-517 (2002)

19. Wyttenbach, T.; Bowers, M.T.: Structural stability from solution to the gas phase: native solution structure of ubiquitin survives analysis in a solvent-free ion mobility-mass spectrometry environment. J. Phys. Chem. B. 115, 12266-12275 (2011)

20. Myung, S.; Badman, E.R.; Lee, Y.J.; Clemmer, D.E.: Structural transitions of electrosprayed ubiquitin ions stored in an ion trap over $\sim 10 \mathrm{~ms}$ to 30 s. J. Phys. Chem. A. 106, 9976-9982 (2002)

21. Skinner, O.S.; McLafferty, F.W.; Breuker, K.: How ubiquitin unfolds after transfer into the gas phase. J. Am. Soc. Mass Spectrom. In press, doi 10.1007/s13361-012-0370-6 (2012)

22. Lomeli, S.H.; Yin, S.; Ogorzalek Loo, R.R.; Loo, J.A.: Increasing charge while preserving noncovalent protein complexes for ESI-MS. $J$. Am. Soc. Mass Spectrom. 20, 593-596 (2009)

23. Lomeli, S.H.; Peng, I.X.; Yin, S.; Ogorzalek Loo, R.R.; Loo, J.A.: New reagents for increasing ESI multiple charging of proteins and protein complexes. J. Am. Soc. Mass Spectrom. 21, 127-131 (2010)

24. Sterling, H.J.; Daly, M.P.; Feld, G.K.; Thoren, K.L.; Kintzer, A.F.; Krantz, B.A. ; Williams, E.R.: Effects of supercharging reagents on noncovalent complex structure in electrospray ionization from aqueous solutions. J. Am. Soc. Mass Spectrom. 21, 1762-1774 (2010)

25. Sterling, H.J.; Cassou, C.A.; Trnka, M.J.; Burlingame, A.L.; Krantz, B.A.; Williams, E.R.: The role of conformational flexibility on protein supercharging in native electrospray ionization. Phys. Chem., Chem. Phys. 13, 18288-18296 (2011)

26. Hogan, C.J. Jr.; Carroll, J.A.; Rohrs, H.W.; Biswas, P.; Gross, M.L.: Combined charged residue-field emission model of macromolecular electrospray ionization. Anal. Chem. 81, 369-377 (2009)

27. Hogan, C.J. Jr., Ogorzalek Loo, R.R.; Loo, J.A.; de la Mora, J.F.: Ion mobility-mass spectrometry of phosphorylase B ions generated with supercharging reagents but in charge-reducing buffer. Phys. Chem., Chem. Phys. 12, 13476-13483 (2010)

28. Catalina, M.I.; van den Heuvel, R.H.; van Duijn, E.; Heck, A.J.: Decharging of globular proteins and protein complexes in electrospray. Chemistry 11, 960-968 (2005)

29. Kaltashov, I.A.; Mohimen, A.: Estimates of protein surface areas in solution by electrospray ionization mass spectrometry. Anal. Chem. 77, 5370-5379 (2005)

30. Kaltashov, I.A.; Abzalimov, R.R.: Do ionic charges in ESI MS provide useful information on macromolecular structure? J. Am. Soc. Mass Spectrom. 19, 1239-1246 (2008)

31. Pagel, K.; Hyung, S.J.; Ruotolo, B.T.; Robinson, C.V.: Alternate dissociation pathways identified in charge-reduced protein complex ions. Anal. Chem. 82, 5363-5372 (2010)

32. Boeri Erba, E.; Ruotolo, B.T.; Barsky, D.; Robinson, C.V.: Ion mobility-mass spectrometry reveals the influence of subunit packing and charge on the dissociation of multiprotein complexes. Anal. Chem. 82, 9702-9710 (2010)

33. Sterling, H.J.; Kintzer, A.F.; Feld, G.K.; Cassou, C.A.; Krantz, B.A.; Williams, E.R.: Supercharging protein complexes from aqueous solution disrupts their native conformations. J. Am. Soc. Mass Spectrom. 23, 191-200 (2012)

34. Hall, Z.; Politis, A.; Bush, M.F.; Smith, L.J.; Robinson, C.V.: Charge-state dependent compaction and dissociation of protein complexes: Insights from ion mobility and molecular dynamics. J. Am. Chem. Soc. 134, 3429-3438 (2012)

35. Robinson, C.V.; Chung, E.W.; Kragelund, B.B.; Knudsen, J.; Aplin, R.T.; Poulsen, F.M.; Dobson, C.M.: Probing the nature of noncovalent interactions by mass spectrometry. A study of protein-CoA ligand binding and assembly. J. Am. Chem. Soc. 118, 8646-8653 (1996)

36. Wu, Q.-Y.; Gao, J.-M.; Joseph-McCarthy, D.; Sigal, G.B.; Bruce, E.; Whitesides, G.M.; Smith, R.D.: Carbonic anhydrase-inhibitor binding: From solution to the gas phase. J. Am. Chem. Soc. 119, 1157-1158 (1997)

37. Liu, L.; Michelsen, K.; Kitova, E.N.; Schnier, P.D.; Klassen, J.S.: Evidence that water can reduce the kinetic stability of protein-hydrophobic ligand interactions. J. Am. Chem. Soc. 132, 17658-17660 (2010)

38. Barrera, N.P.; Di Bartolo, N.; Booth, P.J.; Robinson, C.V.: Micelles protect membrane complexes from solution to vacuum. Science 321, 243-246 (2008)

39. Barrera, N.P.; Isaacson, S.C.; Zhou, M.; Bavro, V.N.; Welch, A.; Schaedler, T.A.; Seeger, M.A.; Miguel, R.N.; Korkhov, V.M.; van Veen, H.W.; Venter, H.; Walmsley, A.R.; Tate, C.G.; Robinson, C.V.: Mass spectrometry of membrane transporters reveals subunit stoichiometry and interactions. Nat. Methods 6, 585-587 (2009)

40. Zhou, M.; Morgner, N.; Barrera, N.P.; Politis, A.P.; Isaacson, S.C.; Matak-Vinkovic, D.; Takeshi, M.; Bernal, R.A.; Stock, D., Robinson, C.V.: Mass spectrometry of intact V-type ATPases reveals lipid binding and the effects of nucleotide binding Science 344, 380-385 (2011)

41. Reimann, C.T.; Valizquez, I.; Tapia, O.: Proteins in vacuo. Denaturation of highly-charged lysozyme studied by molecular dynamics simulations. $J$. Phys. Chem. B 102, 9344-9352 (1998)

42. Chen, L.; Shao, Q.; Gao, Y.-Q.; Russell, D.H.: Molecular dynamics and ion mobility spectrometry study of model beta-hairpin peptide Trpzip 1 . J. Phys. Chem. A 115, 4427-4435 (2011)

43. Baumketner, A.; Bernstein, S.L.; Wyttenbach, T.; Bitan, G.; Teplow, D.B.; Bowers, M.T.; Shea, J.E.: Amyloid beta-protein monomer structure: a computational and experimental study. Protein Sci 15, $420-428$ (2006)

44. Marklund, E.; Larsson, D.S.D.; van der Spoel, D.; Patriksson, A.; Caleman, C.: Structural stability of electrosprayed proteins: temperature and hydration effects. Phys. Chem., Chem. Phys. 11, 8069-8078 (2009)

45. van der Spoel, D.; Marklund, E.; Larsson, D.S.D.; Caleman, C.: Proteins, lipids and water in the gas phase. Macromol. Biosci. 11, 50-59 (2011)

46. Kitova, E.N.; Seo, M.; Roy, P.-N.; Klassen, J.S.: Elucidating the intermolecular interactions within a desolvated protein-ligand complex. An experimental and computational study. J. Am. Chem. Soc. 130, 1214-1226 (2008)

47. Frieman, R.; Larsson, D.S.D.; Wang, Y.; van der Spoel, D.: Molecular dynamics simulations of a membrane protein-micelle complex in vacuo. J. Am. Chem. Soc. 131, 16606-16607 (2009)

48. Knapman, T.W.; Morton, V.L.; Stonehouse, N.J.; Stockley, P.G.; Ashcroft, A.E.: Determining the topology of virus assembly intermediates using ion mobility spectrometry-mass spectrometry. Rapid Commun. Mass Spectrom. 24, 3033-3042 (2010)

49. Hogan, C.J., Jr.; Ruotolo, B.T.; Robinson, C.V.; de la Mora, J.F.: Tandem differential mobility analysis-mass spectrometry reveals partial gas-phase collapse of the GroEL complex. J. Phys. Chem. B 115, 3614-3621 (2011)

50. Hamdy, O.M.; Julian, R.R.: Reflections on charge state distributions, protein structure, and the mystical mechanism of electrospray ionization. $J$. Am. Soc. Mass Spectrom. 23, 1-6 (2012)

51. Hogan, C.J. Jr.; Carroll, J.A.; Rohrs, H.W.; Biswas, P.; Gross, M.L.: Charge carrier field emission determines the number of charges on native state proteins in electrospray ionization. J. Am. Chem. Soc. 130, 6926-6927 (2008)

52. Shelimov, K.B.; Clemmer, D.E.; Hudgins, R.R.; Jarrold, M.F.: Protein structure in vacuo: Gas-phase conformations of BPTI and cytochrome $c$. J. Am. Chem. Soc. 119, 2240-2248 (1997)

53. Bush, M.F.; Hall, Z.; Giles, K.; Hoyes, J.; Robinson, C.V.; Ruotolo, B.T.: Collision cross sections of proteins and their complexes: a calibration framework and database for gas-phase structural biology. Anal. Chem. 82, 9557-9565 (2010) 